\title{
Risk of developing second malignant neoplasms in patients with neuroblastoma: a population study of the US SEER database
}

Hongnan Zhen, Hui Guan, Jiabin Ma, Wenhui Wang, Shen Jing, Zheng Miao, Fuquan Zhang ${ }^{*}$ and Zhikai Liu*

\begin{abstract}
Background: Neuroblastoma is a common extracranial malignant tumor in children. Its main treatment modality is a combination of chemotherapy, radiotherapy, and surgery. Given the advances in chemotherapy regimens and the widespread use of bone marrow transplantation over the decades, there has been improvement in treatment efficacy, which has led to prolonged patient survival. Accordingly, long-term complications have become a growing concern among physicians and patients. This study aimed to analyze the survival rate of patients with neuroblastoma and the risk factors for developing second malignant neoplasms (SMNs).

Methods: The SEER 18 Regs (1973-2015) and SEER 9 Regs (1973-2015) data of the surveillance, epidemiology, and end results (SEER) database of the US National Cancer Institute were adopted for survival and SMN analysis.

Results: The 5-, 10-, and 20-year overall survival rates of patients with neuroblastoma were $67 \%, 65 \%$, and $62 \%$, respectively. Among 38 patients with neuroblastoma who presented with SMNs, those with abdomen as the primary site accounted for the majority (63.2\%), followed by those with thorax (26.3\%) and other sites (10.5\%). SMNs occurred more commonly in non-specific neuroblastoma (incidence: $0.87 \%$ ) than ganglioneuroblastoma (incidence: $0.3 \%$ ). Compared with the general population, the risk of SMN is significantly higher $(\mathrm{SIR}=4.36)$. The risk of developing SMNs was significantly higher in the digestive system $(S I R=7.29)$, bones and joints $(S I R=12.91)$, urinary system $(S I R=23.48)$, brain and other nervous systems $(S I R=5.70)$, and endocrine system $(S I R=5.84)$. Multivariate analysis revealed that the year of diagnosis $(\mathrm{OR}=2.138,95 \% \mathrm{Cl}=1.634-2.797, p<0.001)$ was the only independent risk factor for developing SMNs.
\end{abstract}

Conclusion: This study identifies the risk factor for developing SMNs in patients with neuroblastoma, which could facilitate individualized screening for high-risk patients, to allow early diagnosis and treatment of SMNs.

Keywords: Neuroblastoma, Second malignant neoplasms, Survival rate, Radiotherapy, Chemotherapy

\section{Background}

Neuroblastoma originates from the adrenal medulla or the paravertebral sympathetic nervous system. It is the most common extracranial solid tumor in children.

\footnotetext{
*Correspondence: zhangfuquan3@126.com; liuzk2009@126.com Department of Radiation Oncology, Peking Union Medical College Hospital, Chinese Academy of Medical Sciences \& Peking Union Medical College, No. 1 of Shuaifuyuan Road, Dongcheng District, Beijing 100730, China
}

According to the National Cancer Institute surveys in the United States, the incidence of neuroblastoma was 10.54/1000,000 (between 1975 and 2009). Neuroblastoma is a strongly heterogeneous tumor in children aged $<15$ years. Some tumors can spontaneously disappear without treatment; however, most are insidious and have metastasized to the whole body at the time of diagnosis, with rapid progression ultimately leading to death. International multi-center collaboration of more than 30 years has facilitated an increase in the 5-year survival

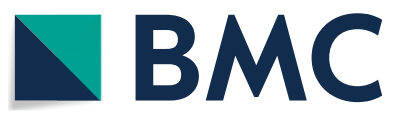

(c) The Author(s) 2021. Open Access This article is licensed under a Creative Commons Attribution 4.0 International License, which permits use, sharing, adaptation, distribution and reproduction in any medium or format, as long as you give appropriate credit to the original author(s) and the source, provide a link to the Creative Commons licence, and indicate if changes were made. The images or other third party material in this article are included in the article's Creative Commons licence, unless indicated otherwise in a credit line to the material. If material is not included in the article's Creative Commons licence and your intended use is not permitted by statutory regulation or exceeds the permitted use, you will need to obtain permission directly from the copyright holder. To view a copy of this licence, visit http://creativecommons.org/licenses/by/4.0/. The Creative Commons Public Domain Dedication waiver (http://creativeco mmons.org/publicdomain/zero/1.0/) applies to the data made available in this article, unless otherwise stated in a credit line to the data. 
rate from $46 \%$ in $1974-1989$ to $71 \%$ in $1999-2004$. This is mainly attributed to the widespread adoption of riskbased stratification of therapy, including decreasing and increasing the treatment intensity for low-to intermediate-risk and high-risk patients [1], respectively, as well as performing autologous stem cell transplantation and metaiodobenzylguanidine (MIBG) treatment. Given the improvement in diagnosis and treatment and the concomitant prolongation of patient survival, long-term complications, represented by second malignant neoplasms (SMNs), have been increasingly attracting attention from physicians and patients. Most patients require postoperative chemotherapy with high-risk patients even needing radiotherapy; contrastingly, few low-risk patients only require postoperative follow-up observation. Radiotherapy was previously considered the main causative factor of SMNs; however, an increasing number of patients who have only undergone surgical treatment without radiotherapy are developing SMNs [2, 3]. This suggests that endogenous factors (mainly genetic and environmental factors) may be involved in the SMN development. In terms of incidence, neuroblastoma is a rare disease and mostly occurs in children and adolescents; it is difficult to study the incidence of SMN through single-center data. Therefore, this study aimed to use data from the Surveillance, Epidemiology, and End Results (SEER) database to investigate the risk factors for developing SMNs after neuroblastoma treatment and explore their impact on patient prognosis.

\section{Methods}

Information of patients with neuroblastoma (including other treatment information) from 1973 to 2015 were retrieved from the SEER database using the software SEER*Stat 8.3.6.1 The inclusion criteria were as follows: (1) patients with ICD-O-3 Hist/behave = neuroblastoma (ICD-O-3 $=9500)$ or ganglioneuroblastoma (ICD-O-3=9490); (2) patients with clear pathological classification and staging; (3) age of diagnosis less than 20 years, and (4) patients with complete followup information. The exclusion criteria were as follows: (1) patients diagnosed by autopsy; (2) primary site of the tumor is the central nervous system (CNS), and (3) patients with incomplete follow-up information. The survival time investigated in this study was the overall survival (OS) time. SMNs are defined as other malignant tumors appearing at least 2 months after neuroblastoma diagnosis. The standardized incidence ratios (SIRs) of SMNs were computed and analyzed using SEER*Stat 8.3.6.1; further, the corresponding 95\% confidence level (CI) was reported. We collected information regarding age at diagnosis, gender, race, pathological type, degree of pathological differentiation, year of diagnosis, primary lesion site, whether radiotherapy was received, whether chemotherapy was received, survival time, and survival status. Authorization was obtained through the SEER website and data were retrieved from the SEER database, with no additional ethical approval being required.

\section{Statistical analyses}

Statistical analyses were performed using SPSS 22.0 (SPSS Inc, Chicago, IL). Statistical significance was set as $p \leq 0.05$. Specifically, the Kaplan-Meier method was used to estimate patient survival time. Univariate and multivariate regression analyses were performed using the Log-Rank test and Cox proportional hazards model, respectively. Independent risk factors for developing SMNs were analyzed using logistic regression. Independent risk factors for death were initially identified using univariate analysis; among them, those with $p \leq 0.1$ were included in the multivariate analysis. The calculation of SIRs are calculated by SEER-stat software (compared with age-matched general population).

\section{Results \\ Clinical characteristics of patients}

This study enrolled 4338 patients, with 3667 patients pathologically-diagnosed with neuroblastoma and 671 with ganglioneuroblastoma. The abdomen $(n=3019)$ was the most common primary site, followed by the chest $(\mathrm{n}=773)$. Males $(52.9 \%)$ and females $(47.1 \%)$ had roughly the same incidence rate. The incidence rates of patients aged $<1$ year, $1-5$ years, and $6-19$ years were $34.4 \%$, $54.1 \%$, and $11.5 \%$, respectively. Table 1 shows the clinical characteristics of all patients with neuroblastoma, including those with SMNs. Among 38 patients with neuroblastoma who presented with SMNs, those with abdomen as the primary site accounted for majority (63.2\%), followed by those with thorax (26.3\%), and other site (10.5\%). SMNs occurred more commonly in non-specific neuroblastoma (incidence: $0.87 \%$ ) than ganglioneuroblastoma (incidence: $0.3 \%$ ). Compared with the general population, the risk of SMN is significantly higher $(\mathrm{SIR}=4.36)$. The risk of developing SMNs was significantly higher in the digestive system ( $\operatorname{SIR}=7.29)$, bones and joints $(\mathrm{SIR}=12.91)$, urinary system $(\mathrm{SIR}=23.48)$, brain and other nervous systems $(\mathrm{SIR}=5.70)$, and endocrine system $(\operatorname{SIR}=5.84)$.

\section{Survival of patients with neuroblastoma}

The 5-, 10-, and 20-year OS rates of the entire neuroblastoma cohort were $67 \%, 65 \%$, and $62 \%$, respectively. Independent risk factors for death were initially identified using univariate analysis; among them, those with $p \leq 0.1$ were included in the multivariate analysis. Univariate analysis identified the following significant 
Table 1 Demographic and clinical characteristics of patients with neuroblastoma, SEER 18

\begin{tabular}{|c|c|c|c|}
\hline \multirow[t]{2}{*}{ Variables } & \multirow[t]{2}{*}{ Categories } & \multicolumn{2}{|l|}{ Cohort (\%) } \\
\hline & & $\begin{array}{l}\text { All NB } \\
\text { patients } \\
(n=4338)\end{array}$ & $\operatorname{SMN}(n=38)$ \\
\hline \multirow[t]{2}{*}{ Sex } & Male & 2294 (52.9) & $19(50.0)$ \\
\hline & Female & 2044 (47.1) & $19(50.0)$ \\
\hline \multirow{3}{*}{$\begin{array}{l}\text { Age at diagnosis } \\
\text { (first primary) } \\
\text { (years) }\end{array}$} & $<1$ & $1493(34.4)$ & $14(36.8)$ \\
\hline & $1-5$ & $2347(54.1)$ & $18(47.4)$ \\
\hline & $6-19$ & $498(11.5)$ & $6(15.8)$ \\
\hline \multirow[t]{5}{*}{ Year of diagnosis } & 1973-1979 & $303(7.0)$ & $5(13.2)$ \\
\hline & 1980-1989 & $506(11.7)$ & $18(47.4)$ \\
\hline & 1990-1999 & $750(17.3)$ & $5(13.2)$ \\
\hline & $2000-2009$ & $1739(40.1)$ & $9(23.7)$ \\
\hline & 2010-2015 & $1040(24.0)$ & $1(2.6)$ \\
\hline \multirow[t]{3}{*}{ Primary Site } & Abdomen & 3019 (70.7) & $24(63.2)$ \\
\hline & Thorax & $773(18.1)$ & $10(26.3)$ \\
\hline & Others & 478 (11.2) & $4(10.5)$ \\
\hline \multirow[t]{2}{*}{ Histologic type } & $\begin{array}{l}\text { Neuroblastoma, } \\
\text { NOS }\end{array}$ & 3667 (84.5) & $36(94.7)$ \\
\hline & $\begin{array}{l}\text { Ganglioneuroblas- } \\
\text { toma }\end{array}$ & $671(15.5)$ & $2(5.3)$ \\
\hline \multirow[t]{3}{*}{ Race } & White & 3410 (79.6) & $30(78.9)$ \\
\hline & Black & $528(12.3)$ & $5(13.2)$ \\
\hline & Other & $348(8.1)$ & $3(7.9)$ \\
\hline \multirow[t]{2}{*}{ Radiotherapy } & Yes & 1085 (25.0) & $19(50.0)$ \\
\hline & No/unknown & $3253(75.0)$ & $19(50.0)$ \\
\hline \multirow[t]{2}{*}{ Chemotherapy } & Yes & $2829(65.2)$ & $28(73.7)$ \\
\hline & No/unknown & 1509 (34.8) & $10(26.3)$ \\
\hline
\end{tabular}

SEER 18, surveillance, epidemiology, and end results program 18; NB, Neuroblastoma; SMN, second malignant neoplasm; NOS, not otherwise specified factors: age $(p<0.001)$, year of diagnosis $(p<0.001)$, histologic type $(p<0.001)$, primary site $(p<0.001)$, chemotherapy $(p<0.001)$, radiotherapy $(p<0.001)$, degree of pathological differentiation $(p<0.001)$, gen$\operatorname{der}(p=0.004)$, and race $(p=0.007)$. SMNs $(p=0.175)$ were not a risk factor for mortality. Factors with $p \leq 0.1$ were included in a Cox proportional hazards model, which identified the following independent risk factors for patient OS: age $(\mathrm{OR}=2.099,95 \% \mathrm{CI}=1.807-2.439$, $p<0.001)$, year of diagnosis $(\mathrm{OR}=5.710,95 \% \mathrm{CI}=3.225-$ $10.110, p<0.001)$, degree of pathological differentiation $(\mathrm{OR}=1.561,95 \% \mathrm{CI}=1.352-1.801, p<0.001)$, primary site $(\mathrm{OR}=1.829,95 \% \mathrm{CI}=1.285-2.602, p<0.001)$, chemotherapy $(\mathrm{OR}=2.864,95 \% \mathrm{CI}=2.048-4.005, p<0.001)$. Radiotherapy $(p=0.08)$ is not a risk factor for mortality.

\section{Risk factors for developing SMNs}

A total of 38 patients were diagnosed with SMNs. The SIR of all the patients was $4.36(95 \% \mathrm{CI}=3.08-5.97$, $p<0.05)$. Univariate analysis showed that the year of diagnosis $(\mathrm{OR}=2.206,95 \% \mathrm{CI}=1.695-2.874, p<0.001)$, radiotherapy $(p=0.001)$, and chemotherapy $(p=0.059)$ were significantly correlated with the incidence of SMNs (Fig. 1). Multivariate analysis showed that the year of diagnosis $(\mathrm{OR}=2.138,95 \% \mathrm{CI}=1.634-2.797, p<0.001)$ was the only independent risk factors for developing SMNs. The result showed that patients with earlier year of diagnosis were more likely to develop SMN. The SIR of SMN gradually increased from 3.44 in the 1970s to 8.14 in the 2000s $(p<0.05)$. Radiotherapy $(p=0.109)$ and chemotherapy ( $p=0.082)$ were not independent risk factors for developing SMNs.

Variable
Age
Gender
Histologic Type
Radiotherapy
Chemotherapy
Year of diagnosis
Grade
Race
Primary site

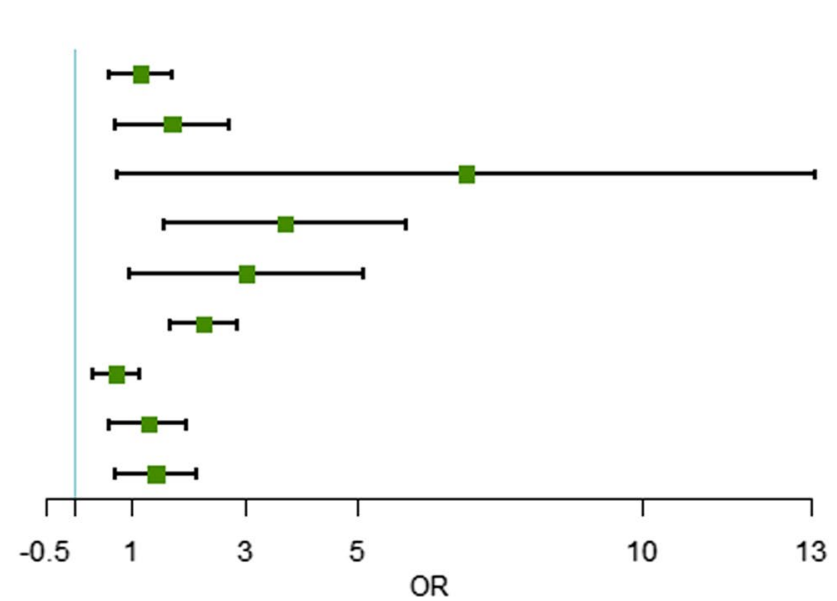

$\begin{array}{cc}\text { OR }(95 \% \mathrm{Cl}) & \text { P value } \\ 1.018 & 0.946 \\ 1.407 & 0.311 \\ 3.13 & 0.117 \\ 3.302 & 0.001 \\ 2.222 & 0.059 \\ 2.206 & <0.001 \\ 0.595 & 0.12 \\ 1.11 & 0.723 \\ 1.244 & 0.432\end{array}$

Fig. 1 The correlationship between risk factors for SMN in neuroblastoma 


\section{Discussion}

This population-based cohort study suggests that the risk of SMNs is higher in patients with neuroblastoma than in the normal population, with the year of diagnosis affecting the incidence of SMNs. There have been several changes in neuroblastoma treatment since the 1970s, including risk-based stratification of therapy (where the treatment intensity for low- to intermediate-risk patients and high-risk patients is decreased and increased, respectively), autologous transplantation, and MIBG treatment. Update of treatment concept and advancement of treatment technology have led to a significant increase in survival rates, which has allowed observation of long-term side effects represented by SMNs in long-term survivors.

Our findings indicate that the year of diagnosis is an independent risk factor for the incidence of SMNs in patients with neuroblastoma. The result showed that patients with an earlier year of diagnosis are more likely to develop SMN, which may be associated with the extension follow-up time and evolution of treatment during the period. High-risk patients are recommended to receive higher-intensity radiotherapy and chemotherapy. Chemotherapy-related hematological SMN usually appeared within a few years after treatment. This has led to a significant increase in the risk of total SMN. However, due to the limitation in the number of cases in the SMN cohort, this view still needs more long-term followup and a larger sample size for verification.

Our study shows that the SMN incidence rate of the entire cohort is $0.88 \%$, which is similar to the incidence of several other neuroblastoma SMN cohorts [2, 4], which is consistent with the results of the Childhood Cancer Survivor Study (CCSS). Studies have shown that the probability of neuroblastoma survivors developing SMNs greatly increases with an increase in survival time. However, the incidence of SMNs does not tend to plateau over time [1, 5-7]. The results of the above studies indicated that the time to SMN occurrence varied according to the pathological type of SMN. The majority of lymphatic and hematopoietic SMNs occur about 5 years after treatment; sarcoma occurs between 5 and 15 years after treatment; and carcinoma usually occurs after 15 years of treatment. With the follow-up time increasing, SMNs are expected to have higher incidence rates.

Our study reveals that radiotherapy is not the independent risk factor for developing SMNs, although most studies have indicated the carcinogenic role of radiotherapy in neuroblastoma treatment [4, 7-12]. Compared to other childhood tumors, radiotherapy doses to treat neuroblastoma are low, and low-dose radiation is more likely to cause radiation-related carcinogenesis. However, the aforementioned conclusions may only apply to traditional radiotherapy dominated by external irradiation.
We found in univariate analysis that radiotherapy was an independent risk factor for SMNs $(p=0.001)$, but multivariate analysis showed that radiotherapy was not a risk factor for SMNs $(p=0.109) .18$ of the 38 patients in our SMN cohort had received radiotherapy (47.3\%), but we were unable to obtain detailed information such as the field of radiotherapy and the site of the SMNs to assess whether SMN occurred in the radiation field. Another reason that may affect the statistical results is that the SMN cohort has a small sample size, which makes it impossible to calculate statistical differences. Some study showed radiotherapy may not play a role in the occurrence of SMN $[3,13]$ mainly because they found that a considerable amount of SMN did not occur in the radiation field, and a small number of SMN patients did not receive radiotherapy; hence, there is insufficient evidence to prove that radiotherapy is implicated in the occurrence of SMN. Another point worth noting is that the wide application of three-dimensional conformal radiotherapy technology (3D-CRT) and intensity-modulated radiotherapy (IMRT) over the past decades has improved the accuracy of treatment and reduced the volume and dose to normal tissues surrounding the radiation field. This may reduce the possibility of SMN occurring around the radiation field. MIBG, which can be broadly considered as a type of radiotherapy, has also been widely applied in neuroblastoma treatment. However, many studies have shown that MIBG is not a risk factor for SMN. Moreover, the post-treatment incidence of SMNs does not vary with the increasing treatment times and radiation dose $[3,13]$.

Our study indicate that chemotherapy is not an influencing factor of SMNs, which is inconsistent with other reports $[4,8]$, and contrary to the current mainstream view. The current mainstream view is that chemotherapy is a risk factor for SMNs, especially cytotoxic drugs represented by alkylating agents and topoisomerase inhibitors, which can cause hematological SMNs such as acute myeloid leukemia (AML). Previously, intermediate-risk patients have a significantly higher risk of developing acute myelogenous leukemia even after receiving chemotherapy with low-dose alkylating agents and topoisomerase inhibitors $[1-3,7]$. However, there is no clear evidence on whether these drugs can cause SMN for sarcoma and cancer. For example some SMN also appeared in patients receiving low-dose chemotherapy [2], and SMN caused by chemotherapy is not dose-dependent, which deserves attention [3]. Since the late 1990s, autologous stem cell transplantation has gradually become the standard of care for high-risk neuroblastoma. Studies have shown that the incidence of SMNs in patients with neuroblastoma receiving autologous stem cell transplantation therapy increased from $1.04 \%$ at 5 posttransplantation years to $2.6 \%$ at 10 post-transplantation 
years. Moreover, the incidence rate tends to continue to increase with an increasing follow-up duration [7]. Notably, low-risk patients usually only receive surgical therapy; however, they still have a significantly higher probability of developing SMNs than expected, which suggests that genetic factors may be crucially involved in the incidence of SMNs [14].

Additionally, patients with relapsed and refractory neuroblastoma are more likely to develop SMNs, which could be associated with genetic factors and higher-intensity chemoradiotherapy [13]. Another example that suggests genes likely playing a role in the development of SMN in patients with neuroblastoma is a SMN cohort had a family history of cancer, but these families did not meet the diagnostic criteria for Li-Fraumeni syndrome (LFS) [1]. LFS is a chromosomal dominant genetic disease, which is related to the mutation of the tumor suppressor gene TP53. LFS can cause various cancers, including breast cancer, brain tumors, sarcomas and other cancers. It usually has a clear family history, and most of it occurs at a young age. The probability of aggressive malignant tumors in high-risk family members before the age of 30 is as high as $50 \%$. Osteosarcoma is a common SMN type after childhood tumor treatment, and is related to radiotherapy and chemotherapy but Ewing's sarcoma is not. A patient with Ewing's sarcoma was found in his SMN cohort, which is a rarer type of SMN pathology, meaning that genetic factors, rather than therapeutic factors such as radiotherapy and chemotherapy, may have caused the occurrence of this SMN. Another study found that multiple gene mutations exist in SMN patients with neuroblastoma [14], but due to the limitation in the number of cases, no statistical significance was found. The above results all indicate that genetic factors cannot be ignored in the pathogenesis of SMN and need to be further studied.

This study has several limitations. First, the treatment information in the SEER database is incomplete; moreover, there is missing key information, including disease stage, radiotherapy dose and genetic information, which impedes deeper analysis, although our study showed that age, year of diagnosis, degree of pathological differentiation, primary site, chemotherapy, and radiotherapy are independent risk factors for the prognosis of patients with neuroblastoma. We assumed that radiotherapy was performed in patients with high-risk disease, which may be a bias in interpreting the results. Neuroblastoma COG (Children's Oncology Group) risk stratification is mainly based on MYCN gene amplification, age, INSS (International Neuroblastoma Staging System) staging and other factors. Because the SEER database lacks MYCN gene amplification, INSS staging information, the current data cannot be used for risk stratification. Second, the
SEER database does not represent the entire US patient population, which may affect the assessment of the actual incidence of SMNs in patients with neuroblastoma. Furthermore, the patient data included in the database cover a large time span with the risk stratification and treatment strategies of neuroblastoma having significantly changed several times since the 1970s. Therefore, the current stratification information and treatment regimens may not match those of past decades, which could introduce bias into the data. Nevertheless, this study revealed some characteristics regarding the incidence of SMNs in patients with neuroblastoma, which provide support for screening of long-term survivors, as well as early detection and intervention of SMNs.

\section{Conclusion}

This study offers data on the SMNs of patients with neuroblastoma, indicating the risk factors for SMNs, which provide insight for neuroblastoma survivors and their physicians during follow-ups.

\begin{abstract}
Abbreviations
SMNs: Second malignant neoplasms; SEER: Surveillance, epidemiology, and end results; SIRs: Standardized incidence ratios; MIBG: Metaiodobenzylguanidine; CNS: Central nervous system; OS: Overall survival; Cl: Confidence interval; CCSS: Childhood Cancer Survivor Study.
\end{abstract}

\section{Acknowledgements}

We wish to thank Shiyu Jiang and Xiaoyin Bai for their encouragement, and Yinjie Tao for his help.

\section{Authors' contributions}

HNZ and ZKL conceptualized and designed the study. FQZ and HG reviewed the literature. JS and JBM collected data and performed statistical analysis. WHW and ZM drafted the manuscript. All authors read and approved the final manuscript.

\section{Funding}

This work was supported by the National Key Research and Development Plan, the Ministry of Science and Technology of the People's Republic of China (Grant Number 2016YFC0105207) and the Non-profit Central Research Institute Fund of Chinese Academy of Medical Sciences (Grant Numbers 2019XK320014). The funding body had no role in the design of the study and collection; analysis, and interpretation of data; and writing of the manuscript.

\section{Availability of data and materials}

The raw data supporting the conclusions of this article will be made available by the authors, without undue reservation.

\section{Declarations}

Ethics approval and consent to participate

Not applicable.

Consent for publication

Not applicable.

Competing interests

The authors declare that they have no competing interests.

Received: 1 October 2021 Accepted: 28 October 2021

Published online: 27 November 2021 


\section{References}

1. Federico SM, Allewelt HB, Spunt SL, Hudson MM, Wu J, Billups CA, et al. Subsequent malignant neoplasms in pediatric patients initially diagnosed with neuroblastoma. J Pediatr Hematol Oncol. 2015;37(1):e6-12. https://doi.org/10.1097/MPH.0000000000000148.

2. Applebaum MA, Vaksman Z, Lee SM, Hungate EA, Henderson TO, London WB, et al. Neuroblastoma survivors are at increased risk for second malignancies: a report from the International Neuroblastoma Risk Group Project. Eur J Cancer. 2017;72:177-85. https://doi.org/10.1016/j.ejca.2016. 11.022

3. Haupt R, Garaventa A, Gambini C, Parodi S, Cangemi G, Casale F, et al. Improved survival of children with neuroblastoma between 1979 and 2005: a report of the Italian Neuroblastoma Registry. J Clin Oncol. 2010;28(14):2331-8. https://doi.org/10.1200/JCO.2009.24.8351.

4. Rubino C, Adjadj E, Guerin S, Guibout C, Shamsaldin A, Dondon MG, et al. Long-term risk of second malignant neoplasms after neuroblastoma in childhood: role of treatment. Int J Cancer. 2003;107(5):791-6. https://doi. org/10.1002/ijc.11455.

5. Garaventa A, Gambini C, Villavecchia G, Di Cataldo A, Bertolazzi L, Pizzitola MR, et al. Second malignancies in children with neuroblastoma after combined treatment with 1311-metaiodobenzylguanidine. Cancer. 2003;97(5):1332-8. https://doi.org/10.1002/cncr.11167.

6. Laverdiere C, Liu Q, Yasui Y, Nathan PC, Gurney JG, Stovall M, et al. Longterm outcomes in survivors of neuroblastoma: a report from the Childhood Cancer Survivor Study. J Natl Cancer Inst. 2009;101(16):1131-40. https://doi.org/10.1093/jnci/djp230.

7. Danner-Koptik KE, Majhail NS, Brazauskas R, Wang Z, Buchbinder D, Cahn $J Y$, et al. Second malignancies after autologous hematopoietic cell transplantation in children. Bone Marrow Transpl. 2013;48(3):363-8. https:// doi.org/10.1038/bmt.2012.166.

8. Travis LB, Demark Wahnefried W, Allan JM, Wood ME, Ng AK. Aetiology, genetics and prevention of secondary neoplasms in adult cancer survivors. Nat Rev Clin Oncol. 2013;10(5):289-301. https://doi.org/10. 1038/nrclinonc.2013.41.

9. Friedman DL, Whitton J, Leisenring W, Mertens AC, Hammond S, Stovall M, et al. Subsequent neoplasms in 5-year survivors of childhood cancer: the Childhood Cancer Survivor Study. J Natl Cancer Inst. 2010;102(14):1083-95. https://doi.org/10.1093/jnci/djq238.

10. Kushner BH, Kramer K, Modak S, Qin LX, Yataghena K, Jhanwar SC, et al. Reduced risk of secondary leukemia with fewer cycles of dose-intensive induction chemotherapy in patients with neuroblastoma. Pediatr Blood Cancer. 2009;53(1):17-22. https://doi.org/10.1002/pbc.21931.

11. Martin A, Schneiderman J, Helenowski IB, Morgan E, Dilley K, DannerKoptik K, et al. Secondary malignant neoplasms after high-dose chemotherapy and autologous stem cell rescue for high-risk neuroblastoma. Pediatr Blood Cancer. 2014;61(8):1350-6. https://doi.org/10.1002/pbc. 25033.

12. Huibregtse KE, Vo KT, DuBois SG, Fetzko S, Neuhaus J, Batra V, et al. Incidence and risk factors for secondary malignancy in patients with neuroblastoma after treatment with (131)I-metaiodobenzylguanidine. Eur J Cancer. 2016;66:144-52. https://doi.org/10.1016/j.ejca.2016.07.017

13. Weiss B, Vora A, Huberty J, Hawkins RA, Matthay KK. Secondary myelodysplastic syndrome and leukemia following 1311-metaiodobenzylguanidine therapy for relapsed neuroblastoma. J Pediatr Hematol Oncol. 2003;25(7):543-7. https://doi.org/10.1097/00043426-200307000-00009.

14. Applebaum MA, Henderson TO, Lee SM, Pinto N, Volchenboum SL, Cohn SL. Second malignancies in patients with neuroblastoma: the effects of risk-based therapy. Pediatr Blood Cancer. 2015;62(1):128-33. https://doi. org/10.1002/pbc.25249.

\section{Publisher's Note}

Springer Nature remains neutral with regard to jurisdictional claims in published maps and institutional affiliations.
Ready to submit your research? Choose BMC and benefit from:

- fast, convenient online submission

- thorough peer review by experienced researchers in your field

- rapid publication on acceptance

- support for research data, including large and complex data types

- gold Open Access which fosters wider collaboration and increased citations

- maximum visibility for your research: over $100 \mathrm{M}$ website views per year

At BMC, research is always in progress.

Learn more biomedcentral.com/submissions 